CLAWAR 2019: 22nd International Conference on

Climbing and Walking Robots and the Support

Technologies for Mobile Machines,

Kuala Lumpur, Malaysia, 26-28 August 2019.

https://doi.org/10.13180/clawar.2019.26-28.08.17

\title{
EXPLORING STRUCTURAL CONTROL OF STIFFNESS IN SYNTHETIC TENDON
}

\author{
C. E. EATON, T. RAMDHONI, and R. S. KARP \\ Colby College Computational Physiology \& Optimization Lab, \\ Department of Computer Science, Colby College, 5853 Mayflower Hill Dr., \\ Waterville, ME 04901, USA \\ E-mail: ceeaton@colby.edu \\ www.colby.edu
}

\begin{abstract}
Tendons shape the performance of biological actuators. The compliance of a tendon in series with a muscle influences that muscle's ability to transmit force, amplify power, or dissipate energy. Similar performance benefits have already been realized in robotic and prosthetic systems with compliant components. With low-cost, commercially available compliant filaments, it is now possible to fine-tune the structural properties of these compliant components during rapid prototyping. However, tailoring the compliance of these parts for diverse tasks such as force transmission, power amplification, or energy dissipation requires fine-grained control of a printed structure's stiffness. Experimental data from printed synthetic tendons are used here to inform a computational model, mapping the space of achievable stiffnesses as a function of cross-sectional area and rest length. Results are verified by comparing the elastic modulus of a single layer of parallel fibers to the filament manufacturer's reported material properties. In multi-layer structures, the alternating orientation of printed fibers from layer to layer was found to significantly influence stiffness, precluding the derivation of this model from the filament's material properties alone. Drawing inspiration from biological tendon, future work will use this mapping to study the influence of custom synthetic tendons on force, displacement, energy, and power transmission pathways between actuators and end effectors in robotic limbs.
\end{abstract}

Keywords: compliance; soft robotics; preflex; 3D printing.

\section{Introduction}

The physiology of animal locomotion showcases the utility of passive compliant mechanisms. ${ }^{1}$ Tendons, in particular, have been shown to fulfill several physiological roles without active control. Tendons make it possible for legged animals to recycle mechanical energy, amplify muscular power, attenuate energy, ${ }^{2}$ and protect muscles from rapid stretch under high loads. ${ }^{3}$ In short, tendon passively shapes the transmission of force and displacement between muscle and the external world.

In-series compliance correlates with muscular efficiency and power transmission, ${ }^{4}$ while correlating inversely with tensile strength ${ }^{5}$ and the transmission of muscular displacement to the skeleton. ${ }^{6}$ These mutually exclusive optimization criteria suggest that performance on the organismal level depends upon variation in tendon properties across anatomical sites, each tuned to suit local functional requirements.

As a passive mechanical link, a tendon's performance is entirely determined by its material and structural properties. Tendons for which the recycling and dissipation of energy are highly desirable tend to be more compliant, while stiffer tendons promote efficient transmission of muscular displacement and work. Accordingly, stiffer tendons are found in anatomical sites such as the proximal limb that place a greater precedence on load bearing and the direct transmission of displacement. More compliant tendons are found in physiological roles for which energy dissipation and spring behaviors are more pressing. ${ }^{6}$ The structural properties of tendon can be tuned through training, ${ }^{5}$ and have been shown to shape the metabolic cost of locomotion. ${ }^{7}$ 
Legged robots have already begun to achieve significant energy savings and reduce the computational overhead of stable locomotion through the incorporation of passive compliant elements. ${ }^{8-11}$ Elastic mechanisms have also been used to amplify actuator power ${ }^{12}$ and safely mitigate large ground reaction forces, "providing the least violent landing" possible. ${ }^{13}$ Going a step further to optimize the structural properties of springs to suit their desired mechanical function has yielded similarly impressive results, reducing the power requirements of a humanoid robot by $47 \% .{ }^{14}$ These biologically inspired success stories motivate further exploration of the extent to which physiological observations of the benefits of compliant materials can be realized in legged robotic systems.

Having shown that compliant elements are capable of promoting the same functions in engineered systems as in physiology, the next steps are to facilitate the rapid prototyping of synthetic tendons with predetermined stiffnesses and to explore the limits of this design space. The present work characterizes the structural control of stiffness in 3D printed synthetic tendons.

With so few compliant filaments available on the commercial market, roboticists interested in a rapid prototyping approach to tendon fabrication are able to exert more control over structural properties than material. Here, synthetic tendons are printed with varying structural characteristics, and their stiffnesses are determined empirically. The relationship between Young's modulus and stiffness is used to present a mathematical mapping between average cross-sectional area of the printed structure and its stiffness.

Soft roboticists will already be familiar with the relationship between Young's modulus (a material property) and stiffness (a structural property), including the correlation between cross-sectional area and stiffness. However, it is interesting to note that while the elastic modulus of all multi-layered printed structures was observed to be relatively constant - as expected of a material property - it was found to be approximately half that of a single layer of parallel fibers. This is likely due to automated inter-layer variation in fiber orientation during the printing process. This phenomenon is consistent with observed variations in the elastic modulus of bone as a function of the orientation of collagen fibers relative to the axis of strain. ${ }^{15,16}$

\section{Methods}

\subsection{Design and fabrication}

All tendons were designed in the open-source $3 \mathrm{D}$ CAD software FreeCAD (version 0.17). ${ }^{17}$ Modeled tendons were sliced in Ultimaker Cura (version 3.4.1), ${ }^{18}$ and printed on a Series 1 Type A Machines 3D printer. ${ }^{19}$ All tendons were printed with $1.75 \mathrm{~mm}$ NinjaFlex ${ }^{20}$ compliant thermoplastic polyurethane (TPU) filament at $220^{\circ} \mathrm{C}$ and an extrusion speed of $15 \mathrm{~mm} / \mathrm{s}$. When printing a tendon, shell thickness was set equal to the thickness of the CAD model and infill density was set to $100 \%$, ensuring that the slicer would not introduce any unfilled pockets other than those explicitly created by the authors in the CAD model.

A fixed length of $115 \mathrm{~mm}$ and width of $25.4 \mathrm{~mm}$ (Figure 1) were chosen to fit between the clamps of the force gauge (Figure 3) while allowing sufficient space for strains in excess of $50 \%$. This length and width were held constant across all tendons, while the thickness varied from $1 \mathrm{~mm}$ to $3 \mathrm{~mm}$.

Tendons were classified in 3 ways based on pocket shape: solid (no pocket), circular, and square. Within a shape classification, tendons were further distinguished by the pockets' cross-sectional area, as shown in Figure 2. Pocket shape and size were uniform within a single tendon, and varied between tendons to test their influence on stiffness.

The dimensions of each printed tendon were measured with calipers after printing, and found to be true to the CAD models' dimensions within the $0.1 \mathrm{~mm}$ tolerance of the calipers. 


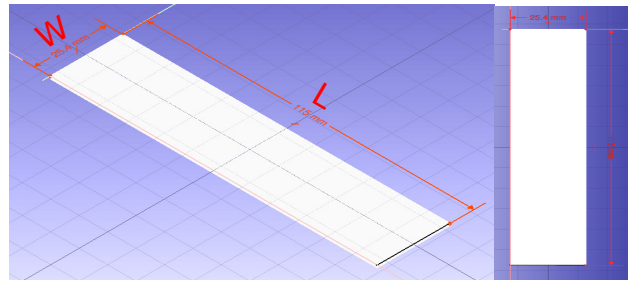

Fig. 1. Outer width $(w=25.4 \mathrm{~mm})$ and length $(l=115 \mathrm{~mm})$ dimensions were kept constant across all 3D printed tendons. Thickness ranged from $1 \mathrm{~mm}$ to $3 \mathrm{~mm}$.

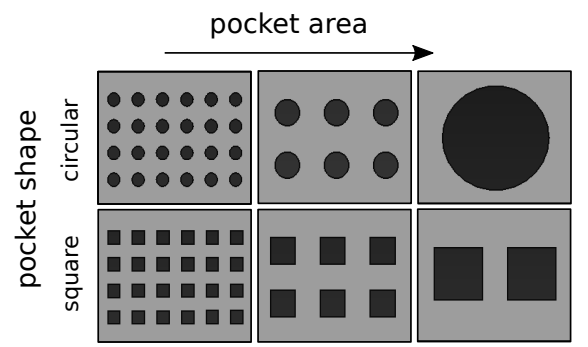

Fig. 2. The shape and cross-sectional area of pockets varied from tendon to tendon, but remained uniform within a single tendon.

The density $\rho$ of each tendon was calculated as a percentage of the volume of a solid tendon (a tendon without pockets) of the same thickness:

$$
\rho=\frac{\left(w * l-a_{p} * n_{p}\right) * t}{w * l * t}
$$

where $w$ is the width, $l$ the length, and $t$ the thickness of the tendon. $a_{p}$ represents the cross-sectional area of an individual pocket, and $n_{p}$ the number of pockets in the tendon.

The average cross-sectional area $a_{t}$ of each tendon was calculated as its solid volume divided by its length:

$$
a_{t}=\frac{\left(w * l-a_{p} * n_{p}\right) * t}{l}
$$

\subsection{Empirical derivation of stiffness}

The tendon was attached to the force gauge with a ribbon grip on each end. Force was produced by stretching the tendon on an FTK100L test stand and measured with a Wagner Instruments FDIX 100 Force Gauge. Force measurements were collected at $10 \mathrm{~Hz}$ using the force gauge and the manufacturer's MESURLite data acquisition software (version 2.0.0).

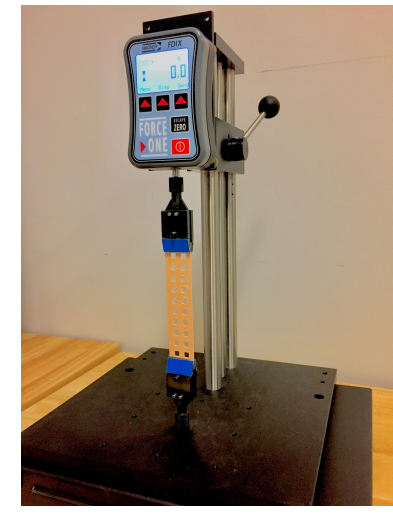

Fig. 3. The experimental setup used to measure forces and lengths during tendon data collection trials. Inter-clamp distance is calculated in OpenCV by tracking the blue gaffers tape affixed to each clamp, while the force gauge uploads a stream of force data to a laptop over USB.

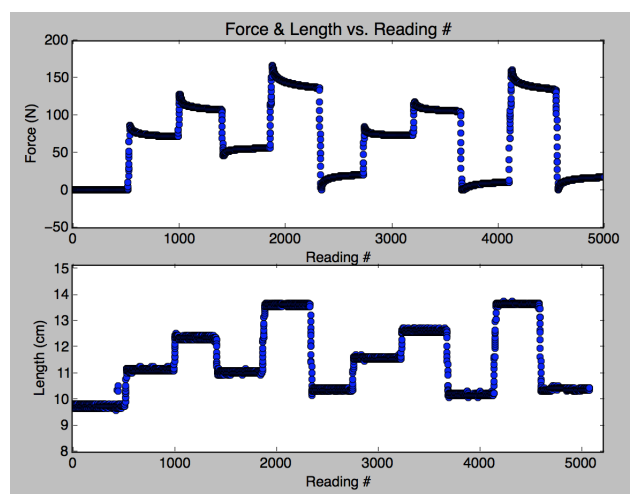

Fig. 4. An example of the force and length data streams collected for a single tendon. Lengths were randomized to alleviate the effects of hysteresis, and each was held steady for several seconds in order to manufacture plateaus in the force and length data streams.

Length was sampled at $10 \mathrm{~Hz}$ using video collected with the webcam of a 13-inch MacBook Pro 2015 as input to a computer vision algorithm written in Python version 2.7.15. 
The OpenCV library ${ }^{21}$ was used to automate detection of blue markers adhered to each clamp. The algorithm calculated distances between the two clamps in centimeters $\left(d_{i}\right)$ by multiplying the initial measured inter-clamp distance in centimeters $\left(d_{0}\right)$ by the ratio of the inter-clamp distance in pixels $\left(p_{i}\right)$ in each frame to the initial inter-clamp distance in pixels $\left(p_{0}\right)$ in the first frame:

$$
d_{i}=d_{0} * \frac{p_{i}}{p_{0}}
$$

Lengths were prescribed by a random number generator in order to mitigate the effects of hysteresis. Maintaining each length for several seconds created easily identifiable plateaus in the force and length data streams, as shown in Figure 4. This facilitated the association of forces from the force gauge's data stream with the corresponding lengths from the computer vision data stream.

A Python script was written to automatically identify the plateaus in the force and length streams. The median value of each force plateau was associated with the corresponding length plateau's median value. A linear regression was then performed to characterize the tendon's force-length relationship. The stiffness of the tendon was calculated as the derivative of this linear regression.

To confirm that this experimental setup can be used to accurately determine the mechanical properties of printed tendons, a trial was conducted on a single layer of parallel printed fibers. Its stiffness $k$ was calculated using the procedure described above. Using the experimentally determined stiffness and the measured tendon dimensions, the material's Young's modulus $(E)$ was calculated (Equation 4 ) as $11.4 \mathrm{MPa}$ - within $5 \%$ of the manufacturer's reported $12 \mathrm{MP}$.

$$
E=\frac{k * l}{a_{t}}
$$

This tendon stretching and plateau matching procedure was repeated to characterize each individual printed tendon.

\section{Results}

Equation 4 describes the well-established relationship between stiffness (a structural property) and Young's modulus (a material property). It can be rewritten to solve for stiffness as

$$
k=\frac{E * a_{t}}{l}
$$

where $k$ is the stiffness of the printed tendon, $E$ its elastic modulus, $a_{t}$ the printed tendon's average cross-sectional area, and $l$ the initial length of the tendon at rest as measured between the force gauge's ribbon clamps. Tendon width was held constant across all 25 multilayer printed tendons, and the resting inter-clamp length remained relatively consistent at $l=9.71 \pm 0.13 \mathrm{~cm}$. It could therefore be predicted that tendon stiffness would correlate with thickness and correlate inversely with pocket area (which decreases average cross-sectional area, as described in Equation 2). This relationship is illustrated in Figure 5.

Stiffness is highly correlated with average cross-sectional area, as shown in Figure 6 . This figure also illustrates the fine-grained control over stiffness that can be achieved through coarse variations in thickness and pocket geometry.

From Equation 5, we would expect the derivative of the stiffness-area relation $\left(\frac{\delta}{\delta a_{t}} k=\right.$ $58.52 * 10^{6} \mathrm{~N} \mathrm{~m}^{-3}$ ) to equal the ratio of the filament's elastic modulus $E$ to the tendons' resting length $l$. This length was relatively consistent at $l=0.0971 \mathrm{~m}$. The elastic modulus 


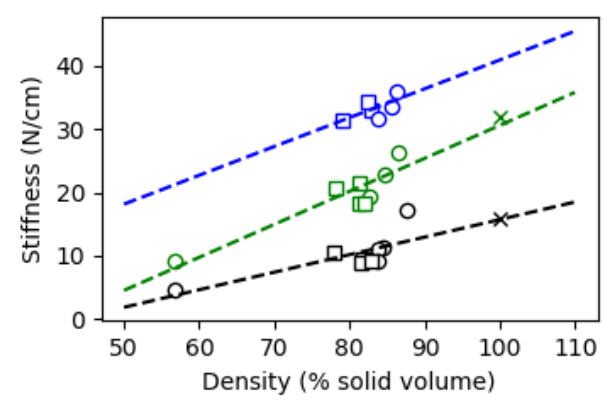

Fig. 5. Tendon stiffness as a function of density. Markers indicate pocket shapes (square $\square$, circular $\circ$, or solid $\mathrm{x}$ ), and tendon thicknesses are differentiated by color (1 $\mathrm{mm}$ black, $2 \mathrm{~mm}$ green, and $3 \mathrm{~mm}$ blue).

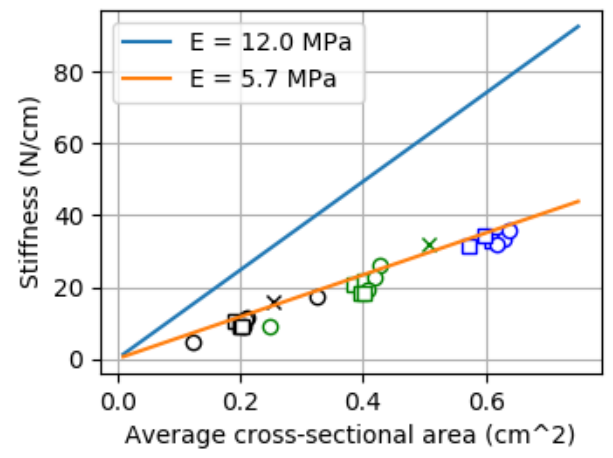

Fig. 6. Tendon stiffness as a function of average cross-sectional area. Markers indicate pocket shapes (square $\square$, circular $\circ$, or solid $x$ ), and tendon thicknesses are differentiated by color $(1 \mathrm{~mm}$ black, $2 \mathrm{~mm}$ green, and $3 \mathrm{~mm}$ blue).

for these printed structures estimated from the linear regression of stiffness-area data is therefore calculated as

$$
E=\frac{\delta}{\delta a_{t}} k * l=5.68 \mathrm{MPa}
$$

This is in close agreement with the average of individual multi-layer printed tendons' empirically determined elastic moduli, which comes to $5.91 \pm 0.77 \mathrm{MPa}$.

However, the elastic modulus of NinjaFlex filament reported by the manufacturer is 12 $\mathrm{MPa}$ - a value verified for a single layer of parallel fibers (11.4 MPa) using the experimental setup presented here. The discrepancy between data collected from a single layer of parallel printed fibers and that of multi-layer printed structures is due to the alternation in fiber orientation from layer to layer. While the fibers within one layer are printed parallel to one another, the slicer software causes adjacent layers to be printed with alternating fiber orientations, likely to enhance stability and strength of the printed structure. Figure 6 illustrates the significant influence of this difference in elastic modulus on the stiffnesses printed structures.

To further investigate the influence of filament orientation on the stiffnesses of multilayer printed structures, 9 additional $1 \mathrm{~mm}$ thick solid tendons (without pockets) were printed in different orientations on the print bed, rotated -30 to +90 degrees relative to the orientation in which all tendons in Figure 6 were printed. All tendons in this batch were $1 \mathrm{~mm}$ thick without pockets (solid volume). The tensile strengths of 6 of these tendons were also determined, using the maximum force recorded by the force gauge before each tendon tore divided by the cross-sectional area of the tendon at rest. (Only 6 tensile strengths were measured to reduce wear and tear on the force gauge.) The orientation of the top layer of printed fibers was observed to vary with tendon orientation on the print bed $\left(\theta_{\text {fiber }}=0.95 *\right.$ $\left.\theta_{\text {tendon }}+46.8 \mathrm{deg}, R^{2}=0.99\right)$. However, print angle $\left(\theta_{\text {tendon }}\right)$ was found to be uncorrelated with both elastic modulus $\left(R^{2}=0.08\right)$ and tensile strength $\left(R^{2}=0.02\right)$. Elastic modulus was found to average $5.73 \pm 1.06 \mathrm{MPa}$, and tensile strength averaged $0.0582 \pm 0.0058 \mathrm{MPa}$. The results (Figure 7) suggest that the alternation of fiber orientation from layer to layer confounds any trends related to the orientation of the tendon on the print bed and the orientation of fibers in the top layer of the print.

Based on these findings, a computational model has been created to map the stiffnesses that can be achieved with the NinjaFlex compliant filament as a function of the printed structure's cross-sectional area $\left(a_{t}\right)$ and rest length $(l)$. This map was generated for both 

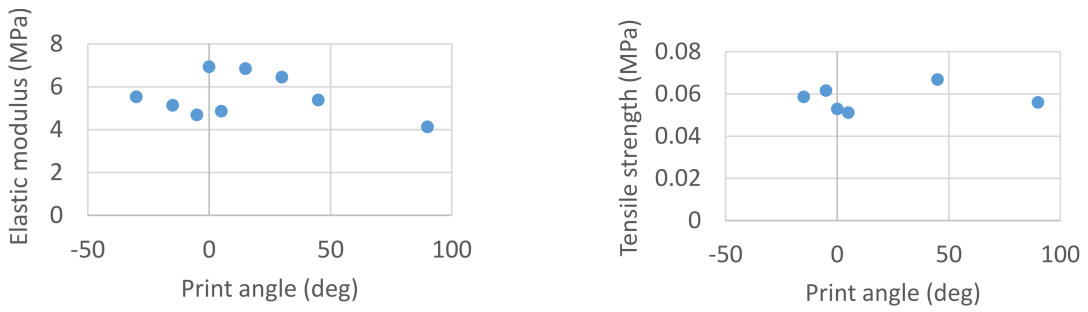

Fig. 7. Although the orientation of the tendon on the print bed has a strong correlation $\left(R^{2}=0.99\right)$ with the orientation of printed fibers in the top layer of the print, this does not seem to influence the elastic modulus or tensile strength of multi-layer printed structures, likely due to alternation of fiber orientations between adjacent layers.

single-layer (Figure 8, $E=12.0 \mathrm{MPa}$ ) and a multi-layer (Figure 8, $E=5.68 \mathrm{MPa}$ ) printed structures.
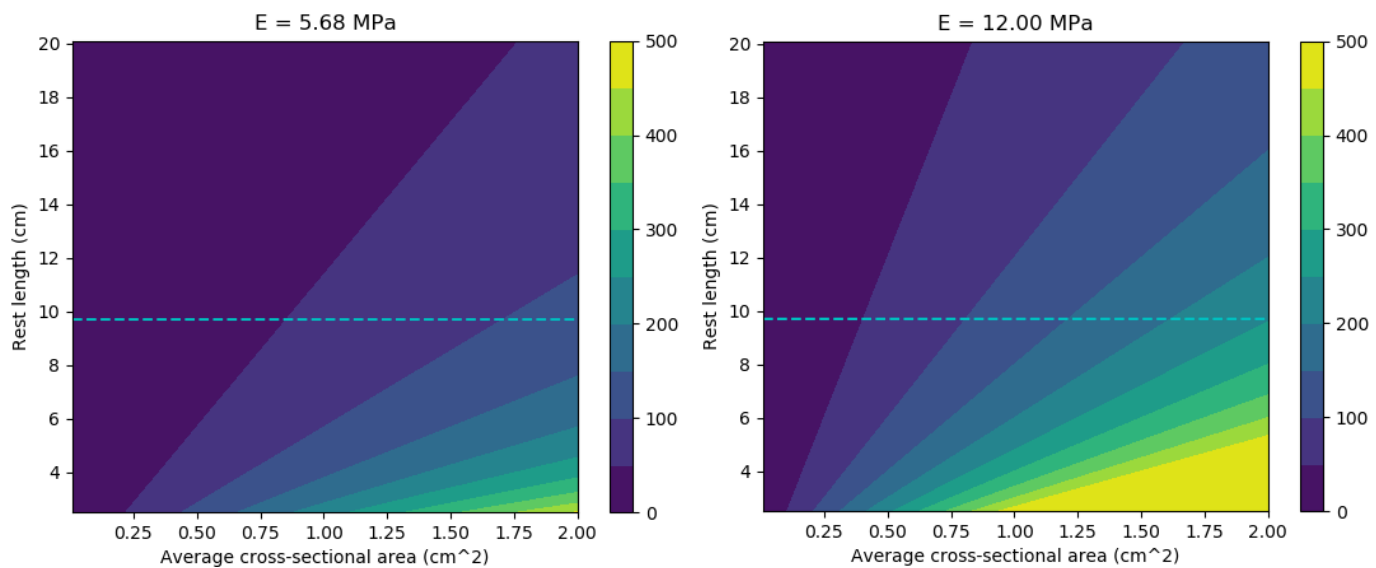

Fig. 8. Response surfaces illustrating the range of stiffnesses that can be achieved in multi-layer $(E=5.68$ $\mathrm{MPa})$ and single-layer $(E=12.00 \mathrm{MPa})$ printed structures by varying cross-sectional area and rest length. The color bar indicates stiffness $k$ in $\mathrm{N} / \mathrm{cm}$. Dashed lines indicate the range of stiffnesses achievable when rest length is held constant $9.71 \mathrm{~cm}$, corresponding to the traces in Figure 6.

\section{Conclusions}

The granularity with which the thickness of a printed structure may be controlled may vary from printer to printer and filament to filament, depending on the minimum viable layer height. Tuning average cross-sectional area through both thickness and pocket area can provide fine-grained control over stiffness (Figure 6) regardless of the achievable layer height.

The 5.68 MPa Young's modulus of multi-layer printed structures is a notable departure from the filament's 11.4 MPa. Although elastic modulus is a material property (rather than structural) and the experimental setup was able to recreate the filament manufacturer's reported elastic modulus to within $5 \%$, the elastic modulus of the printed structures was found to be nearly half that of the filament itself. The stiffness of printed structures could therefore not have been accurately predicted with Equation 5 without first empirically determining the mechanical properties of structures 3D printed with this filament.

This phenomenon is not without precedent. For example, Refs. 15 and 16 observed that the elastic modulus of bone varied with the orientation of its collagen fibers. While printing a structure with a shell thickness in excess of a single layer of printed filament $(\leq 0.1 \mathrm{~mm})$, the 
extruder trajectory generated by the slicing software alternates the orientation of the printed fibers from layer to layer, presumably to promote adhesion and structural integrity. The consistency in elastic modulus among multilayer printed tendons is perhaps more notable than their differences from that of the filament itself.

Although robots have not yet succeeded in recreating the agility and energy efficiency of biological systems, the robotics community's growing understanding of the role of compliant mechanisms in locomotion has led to tangible progress. Physiological studies of locomotion have shown that tendons protect muscle from damage, promote passive self-stabilization, alleviate the neurocomputational overhead of locomotion, and reduce the metabolic cost of transportation. Similarly, in-series compliance has allowed engineered systems to recycle mechanical energy, exhibit passively stable limit-cycle behavior, and free up computational resources previously earmarked for high-frequency state estimation and the micromanagement of end effector placement.

This investigation into the structural control of stiffness in 3D printed synthetic tendon is intended as a preliminary fact-finding mission in the design of increasingly agile, energy efficient legged robots. Drawing inspiration from biological tendon, future work will use the mapping in Figure 8 to study the influence of custom synthetic tendons on force, displacement, energy, and power transmission pathways between actuators and end effectors in robotic limbs.

The ability to tune the stiffness of synthetic tendon also supports the rapid prototyping of prosthetics, with exciting implications for highly customizable, low-cost, open-source prosthetic limbs that could be designed and fabricated by the end user in the comfort of their own home.

\section{Acknowledgments}

The authors would like to thank the office of the Colby College provost, Dr. Margaret T. McFadden, whose support of student research made this work possible. Sincere thanks are also extended to Dr. Gregory C. Pomeroy and Dr. Kevin Rice for the valuable time and resources they have committed to undergraduate research at Colby. Your contributions to

our community extend far beyond the scope of this work, and are deeply appreciated across the college.

\section{References}

1. M. H. Dickinson, C. T. Farley, R. J. Full, M. Koehl, R. Kram and S. Lehman, Science 288, $100(2000)$.

2. T. J. Roberts and E. Azizi, Journal of Experimental Biology 214, 353 (2011).

3. T. J. Roberts and N. Konow, Exercise and sport sciences reviews 41 (2013).

4. G. Lichtwark and C. Barclay, Journal of Experimental Biology 213, 707 (2010).

5. R. E. Shadwick, Journal of applied physiology 68, 1033 (1990).

6. R. Ker, R. M. Alexander and M. Bennett, Journal of Zoology 216, 309 (1988).

7. M. I. V. Orselli, J. R. Franz and D. G. Thelen, Journal of biomechanics 60, 227 (2017).

8. S.-H. Hyon and T. Emura, Advanced Robotics 18, 357 (2004).

9. J. Estremera and K. J. Waldron, The International Journal of Robotics Research 27, 1135 (2008).

10. A. Seyfarth, F. Iida, R. Tausch, M. Stelzer, O. von Stryk and A. Karguth, The International Journal of Robotics Research 28, 257 (2009).

11. M. A. Sharbafi, A. M. N. Rashty, C. Rode and A. Seyfarth, Human movement science 52, 96 (2017).

12. D. W. Haldane, M. Plecnik, J. K. Yim and R. S. Fearing, Science Robotics 1 (2016).

13. N. W. Bartlett, M. T. Tolley, J. T. Overvelde, J. C. Weaver, B. Mosadegh, K. Bertoldi, G. M. Whitesides and R. J. Wood, Science 349, 161 (2015). 
14. N. M. Cahill, T. Sugar, Y. Ren and K. Schroeder, Journal of Mechanisms and Robotics 10, p. 031014 (2018).

15. C. M. Riggs, L. Vaughan, G. P. Evans, L. Lanyon and A. Boyde, Anatomy and embryology 187, 239 (1993).

16. R. Martin and D. Boardman, Journal of biomechanics 26, 1047 (1993).

17. J. Riegel, W. Mayer and Y. van Havre, FreeCAD (version 0.17.13528) (2018), https://www.freecadweb.org/.

18. D. Braam, Cura (version 3.4.1) (2018), https://ultimaker.com/en/products/ultimaker-curasoftware.

19. Type A Machines, Series 1 pro (2017).

20. NinjaTek, Ninjaflex (1.75 mm) (2018), https://ninjatek.com/products/filaments/ninjaflex/.

21. G. Bradski, The OpenCV Library, Dr. Dobb's Journal of Software Tools (2000). 
